\title{
Advanced optical gain materials keep on giving
}

\author{
Qing Zhang ${ }^{1 *}$, Weibo $\mathrm{Gao}^{2^{*}}$ and Qihua Xiong ${ }^{2^{*}}$
}

In the past few decades, there has been a long-standing pursuit to exploring emergent materials with robust optical gain and superior light-matter interacting properties for the development of photonic and optoelectronic devices including but not limited to micro-lasers, single photon emitters, light-emitting-diodes, photo-detectors and so on. Pioneering efforts have been devoted to advanced optical gain materials, covering from classical II$\mathrm{VI} / \mathrm{III}-\mathrm{V}$ semiconductors, emergent two-dimensional semiconducting materials, organic dyes to halide perovskites, which hold great promise for optimizing the device performance and expanding the frontier photonics/optoelectronics. Meanwhile, the scientific and engineering challenges, forging these materials into powerful tools for fundamental sciences and industrial technologies, still remain. The rapid evolution of this area necessitates a highlight of the recent advances and challenges, which is the aim of this timely themed issue on "Optical Gain Materials towards Enhanced Light-Matter Interactions" organized by Science China Materials. This highlight was partly motivated by a symposium organized during the 10th International Conferences on Materials for Advanced Technologies (ICMAT 2019) held in Singapore, co-chaired by all the guest editors.

The wide applicability of optical gain materials highly depends on the intrinsic crystalline and optical quality, and cannot be separated from advanced fabrication techniques. The review by Liu et al. [1] concentrates on the recent studies of various growth methods in halide perovskite semiconductors. Especially, Chen et al. [2] propose the continuous-flow fabrication of doped perovskite nanocrystals in a microfluidic reactor, which enables efficient physical mixing of the precursor ions in the confined micro-channels with stable and closed environment towards high-quality synthesis. Controlled growth of plasmonic heterostructures with a specific composition, morphology, size, and structural symmetry, as well as their optical-related applications are covered by Wang et al. [3]. The progress report by Xiao et al. [4] provides a comprehensive summary of colloidal crystals spheres self-assembly into periodic patterns for optical applications. For typical third-generation semiconductors, Liu et al. [5] overview the development on the design and growth of GaN-based laser diodes.

With advanced optical gain materials and well-designed structures, novel optical and photonic properties resulting from enhanced light-matter interactions can be expected. From a basic scientific perspective, Chen et al. [6] highlight the booming GaAs-based nanowires, especially in the aspect of optical properties and lasing, towards potential infrared laser in integrated optoelectronics. In specific researches, the article by Wang et al. [7] realizes the plasmon-enhanced second-harmonic generation up to 617.7-fold in asymmetric Ag@CdSe hetero-nanorods, enabling high-resolution detection in the biological sensing. An ultrafast formation ( $<100 \mathrm{fs})$ of dihydrogen defects in $\mathrm{YH}_{x} \mathrm{O}_{y}$, which is responsible for the transparentto-opaque photochromic transition with the state stability strongly dependent on the concentration of oxygen, is presented by Cao et al. [8] by using excited-state density functional theory simulation. In the contribution by Pan et al. [9], the strong optical anisotropy of deep-ultraviolet fluorooxoborates with designed possible anionic groups is systematically analyzed to check the influence on birefringence towards developing novel nonlinear optical crystals. Another strategy to enrich the understanding of ideal nonlinear optical crystals, theoretical modeling and simulation, is reported by Lin et al. [10], covering seven potential structures in the deep-ultraviolet region predicted for the first time. For two-dimensional GeS na-

\footnotetext{
${ }^{1}$ Department of Materials Science and Engineering, College of Engineering, Peking University, Beijing 100871, China

${ }^{2}$ Division of Physics and Applied Physics, School of Physical and Mathematical Sciences, Nanyang Technological University, Singapore 637371, Singapore

* Corresponding authors (emails: q_zhang@pku.edu.cn (Zhang Q); wbgao@ntu.edu.sg (Gao W); qihua@ntu.edu.sg (Xiong Q))
} 
noflakes exfoliated experimentally, Jiang et al. [11] observe conspicuous saturated absorption and reverse saturated absorption effects as the incident light are parallel to armchair and zigzag directions of GeS, respectively, revealing the considerable potential for polarizationtunable switching. Moreover, Peng et al. [12] utilize the magneto-photoluminescence measurement to directly reveal the dark exciton in $\mathrm{CsPbBr}_{3}$ perovskite crystals; Xing et al. [13] report the light-induced phase transition and photochromism in two-dimensional $\mathrm{Cs}_{2} \mathrm{PbCl}_{2} \mathrm{I}_{2}$ perovskite crystals, where the newly generated $\mathrm{CsPbI}_{3}$ exhibits a higher stability than the pure cousin. These two papers promote the understanding of halide perovskite-based optoelectronics.

Photonic and optoelectronic applications of advanced optical gain materials, such as miniaturized laser, lightemitting diodes, solar cell, photodetectors, and optical switching, are of intense interest. The continuous-wave pumped laser in semiconductor quantum dots is detailed in the progress report by Zhong et al. [14]. Another contribution by Zhao et al. [15] stresses the organic crystal/Ag nanowire heterostructures and their applications in Boolean logic unit, which paves a hopeful approach into sub-wavelength photonic circuitry. To improve the perovskite solar cell performance, Cheng et al. [16] theoretically predict $\mathrm{PbCl}_{2}$ as a promising candidate for buffer layer in the typical $\mathrm{CH}_{3} \mathrm{NH}_{3} \mathrm{PbI}_{3} / \mathrm{TiO}_{2}$ solar cell structure due to proper bandgap alignment, small lattice mismatch and excellent surface passivation. An extra emphasis by Xing et al. [17] is placed on the high-quality lead-free double perovskites, $\mathrm{Cs}_{2} \mathrm{AgBiBr}_{6}$, as the active region in the solar cell with large grain size $(\sim 0.5 \mu \mathrm{m})$ and tolerable efficiency $(\sim 1.11 \%)$, providing a meaningful guide towards high-efficient non-toxic photovoltaic devices. Furthermore, the applications of van der Waals heterostructures based on two-dimensional layered materials with unique performance are accentuated. $\mathrm{A} \mathrm{Bi}_{2} \mathrm{Se}_{3} / \mathrm{WSe}_{2}$ photodetector investigated by Zhai et al. [18] shows a high detectivity $\left(2.2 \times 10^{10}\right.$ Jones $)$ and a high responsivity $\left(3 \mathrm{~A} \mathrm{~W}^{-1}\right)$ excited by the $1456 \mathrm{~nm}$ nearinfrared source owing to the broken-gap tunneling heterostructure. A $\mathrm{MoS}_{2} / \mathrm{NbS}_{2}$ transistor with $\mathrm{NbS}_{2}$ as the contact electrodes demonstrates a high field-effect mobility $\left(15 \mathrm{~cm}^{2} \mathrm{~V}^{-1} \mathrm{~s}^{-1}\right)$ and a high current on-off ratio $\left(1.8 \times 10^{7}\right)$ and another photodetector with the same structure reveals a high responsivity $\left(1.27 \mathrm{~A} \mathrm{~W}^{-1}\right)$, where the enhanced performance originates from the successful direct epitaxy reported by Gong et al. [19]. A $\mathrm{In}_{2} \mathrm{Se}_{3} / \mathrm{SnSe}_{2}$ photodetector in the work of Yao et al. [20] exhibits an ultralow dark current $(6.3 \mathrm{pA})$, a high detectivity $\left(8.8 \times 10^{11}\right.$ Jones $)$, a high signal-to-noise $\left(>3 \times 10^{4}\right)$ as well as the capability of integration on flexible substrates, where the depletion near the interface and type-II alignment can efficiently suppress the dark current and separate the photo-generated carriers. Finally a CdS/BP photodetector proposed by $\mathrm{Li}$ et al. [21] possesses a record high responsivity up to $9.2 \times 10^{5} \mathrm{~A} \mathrm{~W}^{-1}$ at a low bias voltage of $1.0 \mathrm{~V}$ benefiting from the large light absorption efficiency of CdS.

In summary, this special issue presents some exciting studies that give a flavor of the fascinating scientific and technological developments being carried out in the advanced optical gain materials and related enhanced lightmatter interactions in these systems. Particularly, fabrication, photophysics together with optoelectronic device based on these emergent materials are comprehensively discussed. Finally, we would like to thank the editorial team of Science China Materials for providing the opportunity to edit this special issue. We also sincerely thank all the authors for their outstanding contributions and the referees for their valuable comments that help to improve the articles in this special issue.

Received 1 July 2020; accepted 1 July 2020;

published online 6 July 2020

1 Wang S, Yang F, Zhu J, et al. Growth of metal halide perovskite materials. Sci China Mater, 2020, 63: 1438-1463

2 Lin $\mathrm{P}$, Chen H, Wei Z, et al. Continuous-flow synthesis of doped all-inorganic perovskite nanocrystals enabled by a microfluidic reactor for light-emitting diode application. Sci China Mater, 2020, 63: $1526-1536$

3 Zhong Y, Ma S, Chen K, et al. Controlled growth of plasmonic heterostructures and their applications. Sci China Mater, 2020, 63: 1398-1417

4 Liu J, Zhang X, Li W, et al. Recent progress in periodic patterning fabricated by self-assembly of colloidal spheres for optical applications. Sci China Mater, 2020, 63: 1418-1437

5 Tian A, Hu L, Zhang L, et al. Design and growth of GaN-based blue and green laser diodes. Sci China Mater, 2020, 63: 1348-1363

6 Li H, Chen Y, Wei Z, et al. Optical property and lasing of GaAsbased nanowires. Sci China Mater, 2020, 63: 1364-1381

7 Wu B, Wang PF, Qiu YH, et al. Enhanced second-harmonic generation of asymmetric Au@CdSe heterorods. Sci China Mater, 2020, 63: 1472-1479

8 Chai J, Shao Z, Wang $\mathrm{H}$, et al. Ultrafast processes in photochromic material $\mathrm{YH}_{x} \mathrm{O}_{y}$ studied by excited-state density functional theory simulation. Sci China Mater, 2020, 63: 1579-1587

9 Yang Z, Tudi A, Lei BH, et al. Enhanced nonlinear optical functionality in birefringence and refractive index dispersion of the deep-ultraviolet fluorooxoborates. Sci China Mater, 2020, 63: 1480-1488

10 Kang L, Liang F, Lin Z, et al. Deep-ultraviolet nonlinear optical crystals by design: A computer-aided modeling blueprint from first principles. Sci China Mater, 2020, 63: 1597-1612 
11 Ouyang H, Zhang C, Liu Q, et al. Polarization-tunable nonlinear absorption patterns from saturated absorption to reverse saturated absorption in anisotropic GeS flake and an application of alloptical switching. Sci China Mater, 2020, 63: 1489-1502

12 Shi Z, Muhammad S, Deng L, et al. Magnetic-brightening and control of dark exciton in $\mathrm{CsPbBr}_{3}$ perovskite. Sci China Mater, 2020, 63: 1503-1509

13 Li X, Chen M, Mei S, et al. Light-induced phase transition and photochromism in all-inorganic two-dimensional $\mathrm{Cs}_{2} \mathrm{PbI}_{2} \mathrm{Cl}_{2}$ perovskite. Sci China Mater, 2020, 63: 1510-1517

14 Wang L, Dai G, Deng L, et al. Progress in semiconductor quantum dots-based continuous-wave laser. Sci China Mater, 2020, 63: 1382-1397

15 Lv Y, Xu FF, Wang K, et al. Loss compensation of surface plasmon polaritons in organic/metal nanowire heterostructures toward photonic logic processing. Sci China Mater, 2019, 63: 1464-1471

16 Huang L, Dong H, Huo N, et al. Deep insights into interface engineering by buffer layer for efficient perovskite solar cells: a first-principles study. Sci China Mater, 2020, 63: 1588-1596

17 Zhao D, Wang B, Liang C, et al. Facile deposition of high-quality $\mathrm{Cs}_{2} \mathrm{AgBiBr}_{6}$ films for efficient double perovskite solar cells. Sci China Mater, 2020, 63: 1518-1525

18 Wang F, Luo P, Zhang Y, et al. Band structure engineered tunneling heterostructures for high-performance visible and nearinfrared photodetection. Sci China Mater, 2020, 63: 1537-1547

19 Zhang P, Bian C, Ye J, et al. Epitaxial growth of metal-semiconductor van der Waals heterostructures $\mathrm{NbS}_{2} / \mathrm{MoS}_{2}$ with enhanced performance of transistors and photodetectors. Sci China Mater, 2020, 63: 1548-1559

20 Zheng Z, Chen P, Lu J, et al. Self-assembly $\mathrm{In}_{2} \mathrm{Se}_{3} / \mathrm{SnSe}_{2}$ heterostructure array with suppressed dark current and enhanced photosensitivity for weak signal. Sci China Mater, 2020, 63: 1560-1569

21 Zubair M, Zhu C, Sun X, et al. Record high photoresponse observed in CdS-black phosphorous van der Waals heterojunction photodiode. Sci China Mater, 2020, 63: 1570-1578

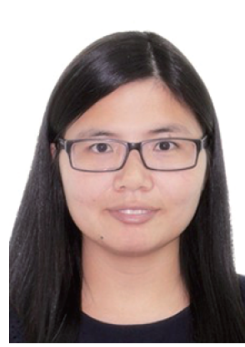

Qing Zhang received her $\mathrm{PhD}$ degree from Tsinghua University in 2011. She is currently an assistant professor in the Department of Materials Science and Engineering of Peking University. Her research interests focus on the lightmatter interaction and optical spectroscopy of low-dimensional semiconductors and their applications in nanophotonics and optoelectronics.

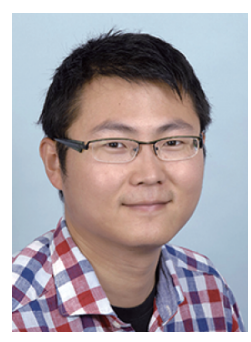

Weibo Gao received his $\mathrm{PhD}$ degree from the University of Science and Technology of China in 2010. He is currently an associate professor in the Division of Physics and Applied Physics in Nanyang Technological University. His research interests include single photon emitters, quantum information application with color centers in wide-band gap material, light-matter interactions and transport properties with two-dimensional materials.

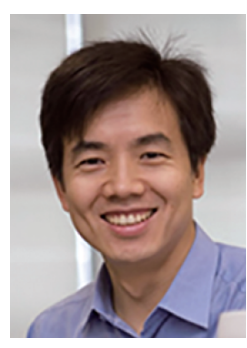

Qihua Xiong received his $\mathrm{PhD}$ degree from Pennsylvania State University in 2006 . He is a leading expert in semiconductor optics and optical spectroscopy. He was elected Fellow of American Physical Society in 2018 and Academician of Asian Pacific Academy of Materials in 2019. He currently serves as Associate Editor for Nano Letters, and international advisory board for many prestigious journals, such as ACS Photonics, Journal of Physics D: Applied Physics, Science China Materials, and Nano Research. 